\title{
Determination of nanoparticles concentration in solution based on Pickering emulsion destabilization analyses
}

\author{
Mariola M. Błaszczyk ${ }^{1}\left[\right.$ tukasz Przybysz $^{1,2}$
}

Received: 7 October 2021 / Accepted: 26 October 2021 / Published online: 8 November 2021

(c) The Author(s) 2021

\begin{abstract}
The dynamic development of nanotechnology research has contributed to the fact that various types of nanoparticles are increasingly used on a large scale both for medical and biological purposes, but above all in many industrial fields. Such a wide application of nanoparticles is often connected with the need to estimate their characteristic parameters, such as size, size distribution or concentration. Existing instruments are usually quite expensive and not always available. Therefore, other cheaper and simpler methods based on analytical techniques are sought. In this paper, we have proposed a method to estimate the concentration of nanoparticles in solutions based on destabilization analyses of Pickering emulsions produced with their use. The fact of mutual relationship between emulsion concentration, nanoparticle concentration and emulsion stability was used here. The study was carried out using silica nanoparticles. It was presented how to apply the method and what are its limitations. Moreover, an example of its application for the determination of nanoparticle concentration in an unknown sample, obtained after analysis of the permeability of membranes in diffusion chambers, has been presented. The method can become a useful alternative for the determination of nanoparticle concentration in solution in places where no specialized equipment is available.
\end{abstract}

Keywords Nanoparticles $\cdot$ Nanoparticle concentration $\cdot$ Pickering emulsion $\cdot$ Emulsion destabilization

\section{Introduction}

The tremendous development of nanotechnology in the last decade has led to the widespread use of various types of nanoparticles in medicine, biology and industry. According to the European Commission, nanoparticles are small particles ranging in size from 1 to $100 \mathrm{~nm}$ (Commission Recommendation 2011/696/EU). They are most commonly used for medical purposes as disinfectants (Sudagidan et al. 2021). Continuous scientific progress shows their use as fluorescent biological labels (Yu et al. 2021a), drug carriers (Jin et al. 2020), they are also used for protein detection (Shahbazi et al. 2021), separation and purification of

Mariola M. Błaszczyk

mariola.blaszczyk@p.lodz.pl

1 Faculty of Process and Environmental Engineering, Department of Chemical Engineering, Lodz University of Technology, 213 Wolczanska St., 90-924 Lodz, Poland

2 Institute of Agriculture and Food Biotechnology, Refrigeration Technology and Technique Department, 84 Al. Marszałka J. Piłsudskiego, 92-202 Lodz, Poland biological molecules and cells (Eivazzadeh-Keihan et al. 2021), in tissue engineering (Wang et al. 2021) and many others (Khalegh and Qaderi 2019). However, on a large scale, nanoparticles are used in industry. Carbon nanotubes are commonly added to various construction materials to improve their endurance properties (Al-Thobity and Gad 2021). Nano-fuels are used in environmental processes (Kegl et al 2021). Applications of metal oxides in flexible solar cells are being developed (Kumar et al. 2020; Sheikholeslami and Ehteshami 2020), as well as for purification of polluted water (Yu et al. 2021b). In the cosmetics industry, mineral nanoparticles-such as titanium oxide-are used in sunscreens because of the low longterm stability offered by conventional chemical UV protection (Kaur et al. 2021). One very modern technology is the Sol-Gel process, which produces a solid material from nanoparticles (Zheng and Boccaccini 2017). Nanoparticles are nowadays widely used as components of processing slurries. Operation with traditional machining slurries introduces contamination to the environment, because they usually consist of toxic or polluted ingredients (Liao et al. 2021). The silica nanoparticles are employed to 
overcome this challenge, by development of novel slurries to polish copper (Zhang et al. 2019), sapphire (Zhang et al. 2021), titanium (Zhang et al. 2018) and nickel alloys (Zhang et al. 2020). Nanoparticles and nanotechnology have also been used for the development of innovative machining approaches and diamond wheels (Zhang et al. $2015,2017)$. With these widely used unique materials, high-performance devices are fabricated (semiconductors, microelectronic and optoelectronic equipment) with the use of developed slurries, approaches and diamond wheels (Zhang et al. 2012). Which is extremely difficult to be manufactured by traditional technologies and production processes. The key feature is that the developed novel methods and slurries eliminate the pollution to the environment, which is a significant contribution of the conventional manufacturing (Wang et al. 2018; Xie et al. 2020).

Considering the use of nanoparticles on such a large scale in various fields of life, it is necessary to develop methods and techniques to accurately determine their parameters such as their size, shape, size distribution and concentration (Park et al. 2020; Pellequer et al. 2021). Modern devices based on advanced laser induced breakdown spectroscopy techniques have been designed (Menneveux et al. 2015). The well-known techniques are DLS (Dynamic Light Scattering) (Brar and Verma 2011; Yaraki and Tan 2020) or NTA (Nanoparticle Tracking Analysis) (Gallego-Urrea et al. 2011) and their numerous modifications (Alasonati et al. 2021) based on which it is possible to determine precisely the size of even very small particles. However, it is quite problematic to determine the concentration of nanoparticles in solution, especially when the concentration is low and the sample is characterized by high clarity. The most well-known techniques for determining the concentration of nanoparticles are gravimetric analysis, bicinchoninic acid assay, Bradford assay and alkaline hydrolysis combined with UV spectroscopy (Khramtsov et al. 2021). Single particle-inductively coupled plasma-mass spectrometry is also used (Chao et al. 2020). Less common, but also encountered methods used to determine the concentration of nanoparticles are based on turbidimetric techniques (Tchami et al. 2019).

Despite the relatively high accuracy of the mentioned methods, they are all characterized by a high degree of complicatedness and the use of complex components. This makes the devices, in which these techniques are used, expensive and difficult to operate. Considering the scale on which various types of nanoparticles are currently used, simpler and cheaper methods for estimating the concentration of nanoparticles in solution are sought. Such methods could be based on certain standards, which, when compared with the analyzed sample, would give satisfactory accuracy. Of course, it is not possible to create one method and one standard for all types of nanoparticles, so each type must be considered separately.
An important feature of a rather large group of nanoparticles is their ability to form so-called Pickering emulsions (Ramsden 1903; Pickering 1907; Metin et al. 2014). Emulsions are systems composed of two phases-oil and water, where one phase is dispersed in the other, while the stability of such a system is due to the presence of a third component-a stabilizer (emulsifier) (Becher 1957). In the case of Pickering emulsions, the role of stabilizer is fulfilled by nanoparticles. Among nanoparticles having the ability to stabilize emulsions, there are numerous types of inorganic particles including silica, clay, and hydroxyapatite (Hap), as well as some organic particles, can effectively serve as Pickering emulsifiers (Yang et al. 2017). The nanoparticles have the ability to absorb on the interfacial surface of the inner phase droplets, forming a coating on the surface that counteracts the droplet coalescence process (Low et al. 2020; du Sorbier et al. 2015). The process of coalescence involves the merging of individual droplets into one larger droplet, which then, due to density differences, leads to creaming or sedimentation, which are the clustering of droplets near the surface or at the bottom of the vessel. As a result, phase separation occurs and the emulsion loses its properties (Simovic and Prestidge 2004).

The stability of Pickering emulsions is dependent on the emulsion formulation, ambient conditions but most importantly on the concentration of nanoparticle addition (Chevalier and Bolzinger 2013). There are many papers in the literature on the stability of Pickering emulsions, where different factors affecting the phase separation rate are studied (Ashby and Binks 2000; Binks and Lumsdon 2001; Cunha et al. 2014; Binks and Yin 2016; Calabrese et al. 2018; Dammak and Sobral 2018; Lebdioua et al 2018; Bao et al. 2019). All these works prove a direct relationship between emulsion concentration, nanoparticle concentration and destabilization rate. In this work, this reciprocal relationship was used to develop an indirect method for determining the concentration of dilute nanoparticle solutions. This method was based on data obtained for silica nanoparticles; however, similar estimates can be made for other nanoparticles having the ability to form Pickering emulsions. This method can be used to determine the concentration of nanoparticles in aqueous solutions obtained during particle permeability tests through filters in diffusion chambers, without the need for specialized equipment.

\section{Experimental part}

The basis for the development of a method for determining nanoparticle concentrations in solution was a thorough analysis of the stability of Pickering emulsions, produced using the studied nanoparticles as a stabilizer. Therefore, series of oil-in-water emulsions stabilized with 
silica nanoparticles were created and then subjected to detailed stability studies. The internal phase of the emulsion was the mineral oil (Orlen Platinum Classic Life +), the viscosity of which was $48.12 \pm 0.05$ and the density equaled $886.25 \pm 0.15 \mathrm{~kg} / \mathrm{m}^{3}$ at $22{ }^{\circ} \mathrm{C}$. The aqueous phase was distilled water. Hydrophilic silica nanoparticles with an average particle diameter of $9 \mathrm{~nm}$ were used as a stabilizer. The procedure for creating the emulsion was following: the measured amount of silica was mixed with the aqueous phase and subjected to ultrasound $(40 \mathrm{kHz})$ for $300 \mathrm{~s}$. Then, this mixture was combined with the oil phase and submitted to emulsification, using a highspeed mixer with a rotational frequency of $20,000 \mathrm{~s}^{-1}$ for $180 \mathrm{~s}$. The emulsion prepared in this way was immediately subjected to nephelometric analysis and droplet size distribution analysis. Droplet size distribution was analyzed using Anton Paar Particle Size PSA Analyzer 1190. PSA equipment through laser diffraction technology can determine the particle size and size distribution. Laser diffraction measures particle size distributions by measuring the angular variation in intensity of light scattered as a laser beam passes through a dispersed particulate sample. The angle of the laser beam and particle size have an inversely proportional relationship, where the laser beam angle increases as particle size decreases and vice versa. The instrument allowed the determination of the size diameter distributions of all the systems studied and the determination of the mean diameter. Figure A1 (Appendix) presents the mean diameter values for all the systems studied. To determine the changes in the distribution of the oil droplets diameter, the samples were analyzed immediately after forming the emulsion $(t=0 \mathrm{~s})$ and after $1 \mathrm{~h}(t=3600 \mathrm{~s})$. This made it possible to determine if and to what extent the droplet coalescence process occurs. The nephelometric analysis was performed with the use of Turbiscan LabExpert by Formulation. In this device, the measuring cell containing the emulsion sample was scanned with a light beam. Using detectors, the amount of reflected light (BS) and light transmitted (T) through the beam was measured. Scanning took place every $30 \mathrm{~s}$ for an hour, which allowed to observe changes in the emulsion structure over time along the sample. Emulsions with different concentrations of the oil phase $\varphi_{\mathrm{e}}$ and different concentrations of nanoparticles $\phi_{z}$ were analyzed. The concentration range of the internal phase was between 0.10 and 0.60 vol. The addition of silica nanoparticles was defined as the weight concentration in relation to the water phase. The addition of silica nanoparticles ranged from 0.1 to $4.5 \%$ by weight. Destabilization studies of the resulting systems were performed for each sample for $1 \mathrm{~h}$. The temperature at which the measurements were carried out was $22{ }^{\circ} \mathrm{C}$.

\section{Analysis of the destabilization process}

The proposed method for determining the concentration of nanoparticles in solution is based on relating the amount of nanoparticles, the concentration of the emulsion to the rate of the destabilization process. Therefore, in the first step, a detailed description of the disintegration process of the created systems will be presented. Prepared under strictly defined conditions oil-in-water emulsions, stabilized with silica nanoparticles, were analyzed during one hour from the time of their formulation. At this time, the kinetics of the creaming process were observed. An exemplary photo of the tested emulsions after preparation and after $1 \mathrm{~h}$ is shown in Fig. 1.

As can be seen visually, from the pictures (Fig. 1), after an hour the creaming process takes place, that is, under the influence of gravity, oil phase drops collect at the top of the sample, while the water phase remains at the bottom of the sample. The kinetics of the creaming process in time can be represented by Fig. 2, where the blue color corresponds to the clear zone (water) and the orange color to the reflecting light zone (oil drops). However, it can be observed that phase separation was finished after this time.

This process can be studied also over time by analyzing the light transmitted through the sample using a Turbiscan LabExpert device. The sample was scanned for an hour, at intervals of $30 \mathrm{~s}$. As a result, diagrams of the transmitted light $\mathrm{T}$ and reflected light $\mathrm{BS}$ versus sample height for subsequent, successive scans were obtained, as shown in Fig. 3. The first scans were represented in blue color, the next scans moved towards red color.

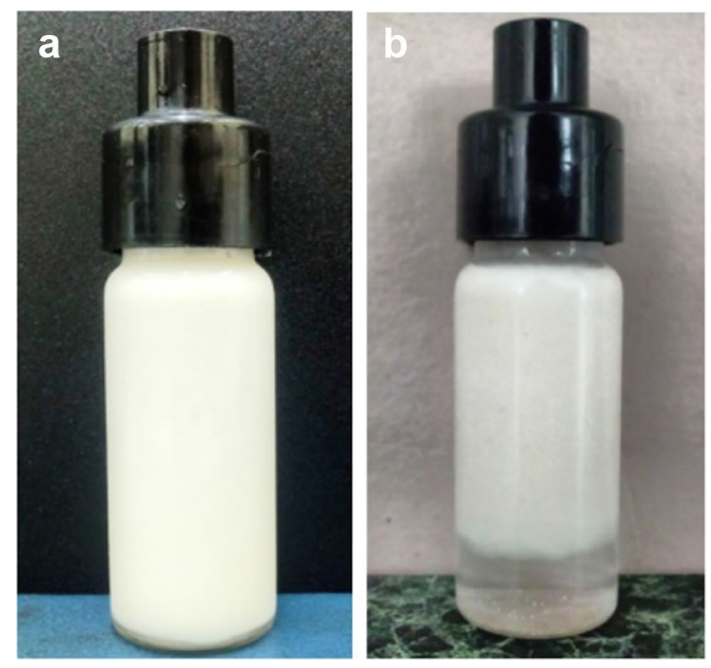

Fig. 1 Pictures of emulsion samples stabilized with silica nanoparticles a immediately after formation $(t=0 \mathrm{~s}), \mathbf{b}$ after $1 \mathrm{~h}(t=3600 \mathrm{~s})$ $\varphi_{\mathrm{e}}=0.30, \phi_{z}=0.67 \%$ 
As can be seen from Fig. 3 in the initial time (blue lines), the values of transmitted and reflected light were constant along the height of the sample. However, for subsequent scans, the reflected light values began to decreased at the bottom of the sample, while the transmitted light values increased. The curves of the relationship $\mathrm{T}$ and BS versus height determined two zones. The first represents the height at which low BS values and higher $\mathrm{T}$ are recorded, which refers to the separated aqueous phase. The second one is represented by high $\mathrm{BS}$ and zero $\mathrm{T}$ values, which refer to the layer of cream created by the oil droplets of the emulsion. As can be seen, the thicknesses of these zones change over time towards an increase in the water zone and a decrease in the emulsion zone. Changes in the height of the water zone over time for emulsions with an initial concentration of 0.25 vol. using different concentrations of silica nanoparticles are presented in Fig. 4.

As can be seen from the graph in Fig. 4, the thickness of the water phase changes with time depends on the concentration of silica nanoparticles. For emulsions produced at low $\phi_{z}$ concentrations, the thickness of the oil phase increases rapidly and reaches high values, when compared to emulsions prepared with a large addition of nanoparticles. At the final stage of measurements, the increase in thickness lowered and reached final height $\left(h_{\max }\right)$. In the case of an emulsion with an oil phase of 0.25 vol. at a
Fig. 2 Creaming process of emulsion in time $-\varphi_{\mathrm{e}}=0.30$, $\phi_{z}=0.67 \%$ silica

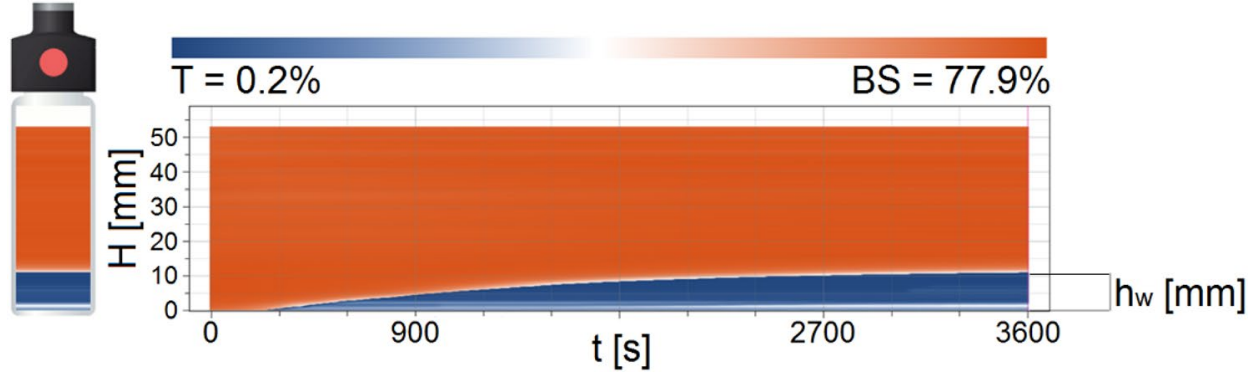

Fig. 3 Changes in light transmittance (T) and reflected light (BS) relative to the sample height for subsequent scans $-\varphi_{\mathrm{e}}=0.30, \phi_{z}=0.67 \%$ silica, during $1 \mathrm{~h}$

Fig. 4 Changes in the water phase thickness over time at different concentrations of silica nanoparticles additions-emulsion $0.25 \mathrm{vol}$
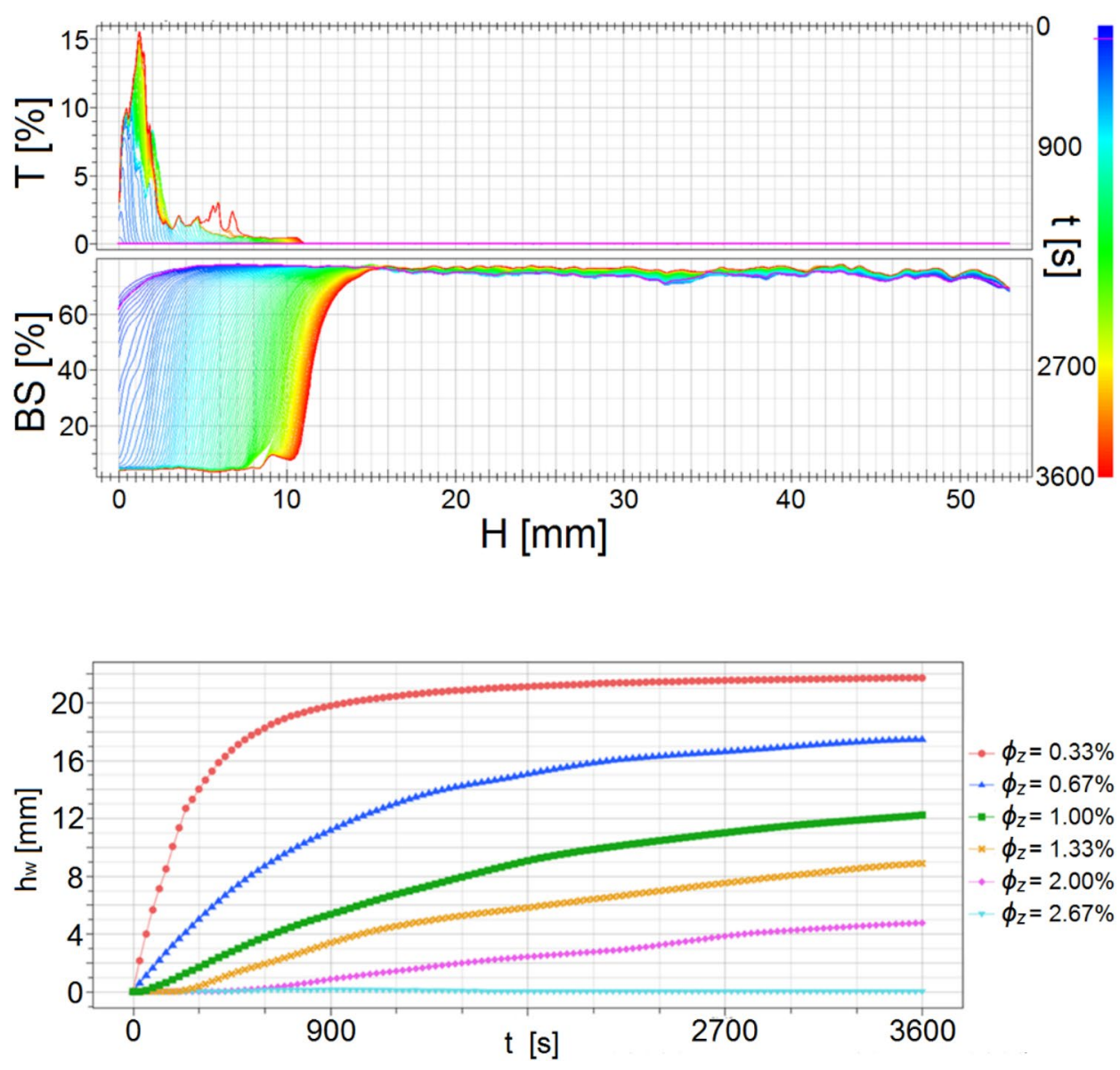
nanoparticle concentration of $\phi_{z}$ equal $2.67 \%$, the water phase was not observed, the thickness water equaled zero, which means that the emulsion remained stable for the duration of the measurements.

\section{Analysis of creaming process velocity changes}

By tracking changes of concentration in emulsion samples over time, it is important to know the process velocity changes over time. The velocity of the destabilization process was calculated from the dependence:

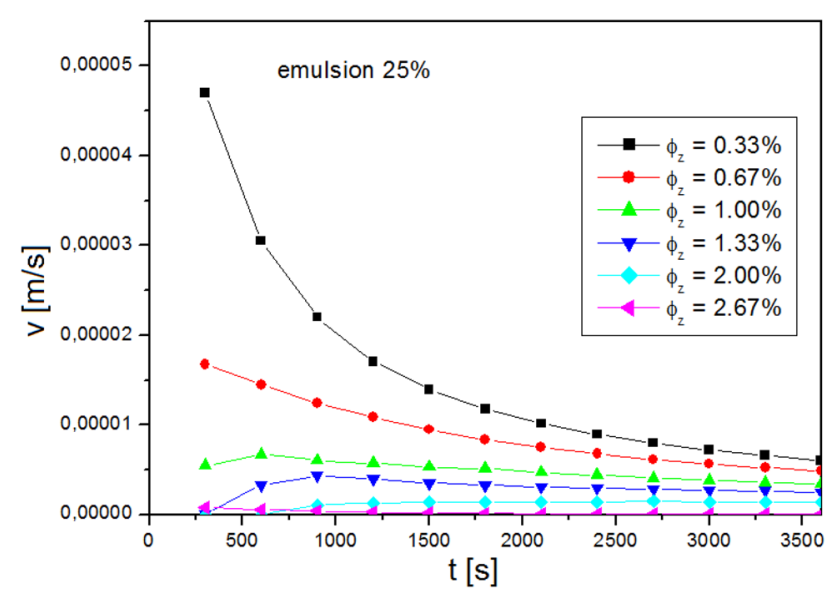

Fig. 5 The average velocity of creaming process versus time-at different concentrations of silica nanoparticles additions-emulsion 0.25 vol

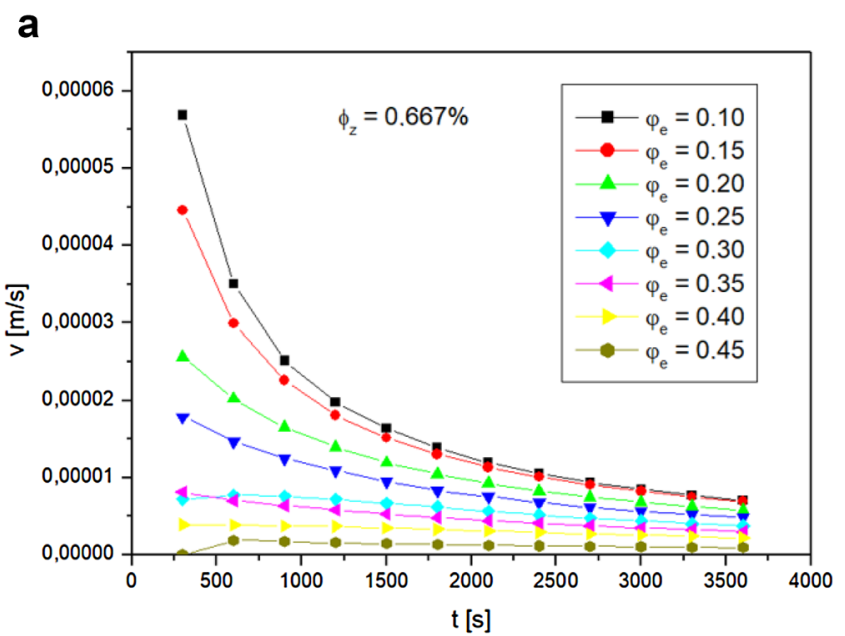

$v=\frac{h_{w i}}{t_{i}}$,

where $t_{i}$-measurement time from the beginning of the destabilization process to time $i$ (s), $h_{w i}$-thickness of water phase at any given time $i(\mathrm{~m})$.

For emulsions with an oil phase share of 0.25 vol. at different concentrations of silica nanoparticles, the graph of average velocity versus time, is shown in Fig. 5.

As can be seen in Fig. 5, the average process velocity decreased over time. Finally it reached the constant value. For the emulsion with the smallest addition of stabilizer, the velocity in the initial stage of the process took large values, but it stabilized over time. In the case of the emulsion at which no creaming process took place (addition of $\phi_{z}=2.67 \%$ silica/water), the velocity equaled zero. The smaller nanoparticles addition, the higher velocities noted. It should be remembered that the rate of destabilization of such emulsions does not depend only on the addition of nanoparticles (stabilizer) but also on the concentration of the internal phase of the emulsion itself. Therefore, destabilization studies were carried out at different volume fractions of the emulsion internal phase. Figure 6 presents graphs of changes in average velocity over time for different concentrations of emulsion inner phase $\left(\varphi_{e}\right)$ at the same concentration of stabilizer- $\phi_{z}=0.67 \%$-for Fig. $6 \mathrm{a}$ and $\phi_{z}=1.33 \%$-for Fig. 6b.

Based on the graphs presented in Fig. 6, it can be observed that the velocity of the process strongly depends on the proportion of the internal phase of the emulsion. The less concentrated the emulsion, the higher velocity recorded. At low concentrated emulsions, in the initial stage of the process, the velocities reached high average values, and as the process decreases, they achieve a constant value. For

b

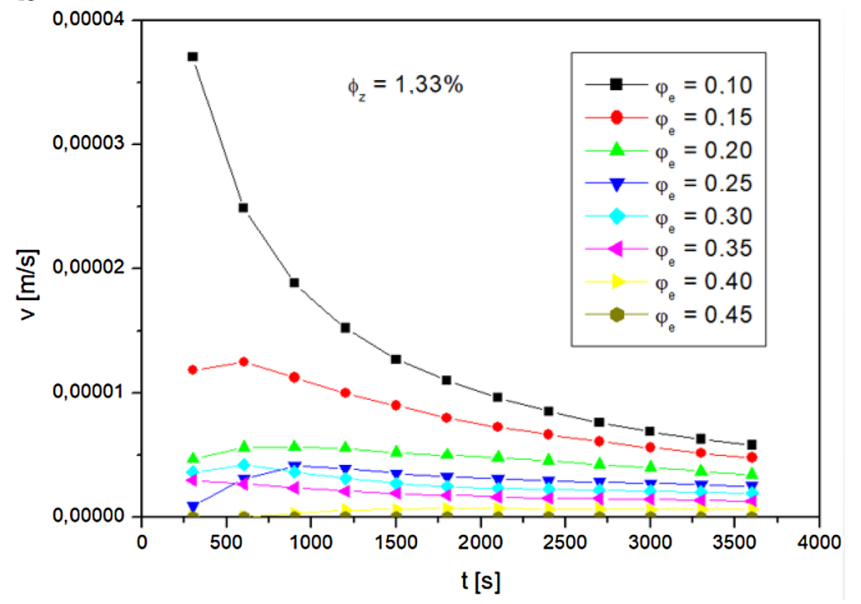

Fig. 6 The average velocity of creaming process versus time at different initial concentrations for $\mathbf{a} \phi_{z}=0.67 \%, \mathbf{b} \phi_{z}=1.33 \%$

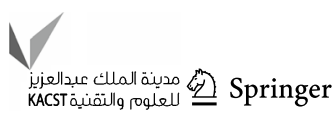


emulsions with higher concentrations, changes in velocity over time were small, and for stable emulsions $\left(\varphi_{\mathrm{e}}=0.45\right.$, $\phi_{z}=1.33 \%$ ) were zero. Based on the study, it can be concluded that the rate of destabilization is closely related to both the concentration of nanoparticles and the concentration of the emulsion itself, and it is possible to quantitatively capture this relationship.

\section{Determination of a standard curve for estimating the concentration of nanoparticles in solution based on the average destabilization rate}

Although changes in destabilization velocity during the process are important, it is more useful to determine the average velocity $v_{\mathrm{m}}$ at a given time. To determine this, the height $h_{\mathrm{w}}$ (Fig. 4) was read for the final time (i.e. the value of the aqueous phase thickness recorded for the time $3600 \mathrm{~s}$ ) and divided by the total time of the process (that is, for $3600 \mathrm{~s}$ ). This allowed for the average destabilization velocity to be compared with the concentration of the nanoparticles and the concentration of the emulsion itself. Figure 7 presents the dependence of the silica nanoparticle concentration $\phi_{z}$ on the destabilization velocity $v_{\mathrm{m}}$ for three different concentrations of the internal emulsion phase $\varphi_{\mathrm{e}}$. From this graph, it can be observed that this velocity decreases with increasing concentration $\phi_{z}$, in other words, an increase in the concentration of silica nanoparticles causes a slower velocity of the destabilization process, noted after an assumed time $(1 \mathrm{~h})$. However, the trend is different for different concentrations of the inner phase of the emulsion. For an emulsion with a concentration of 0.1 vol., this decrease is milder than

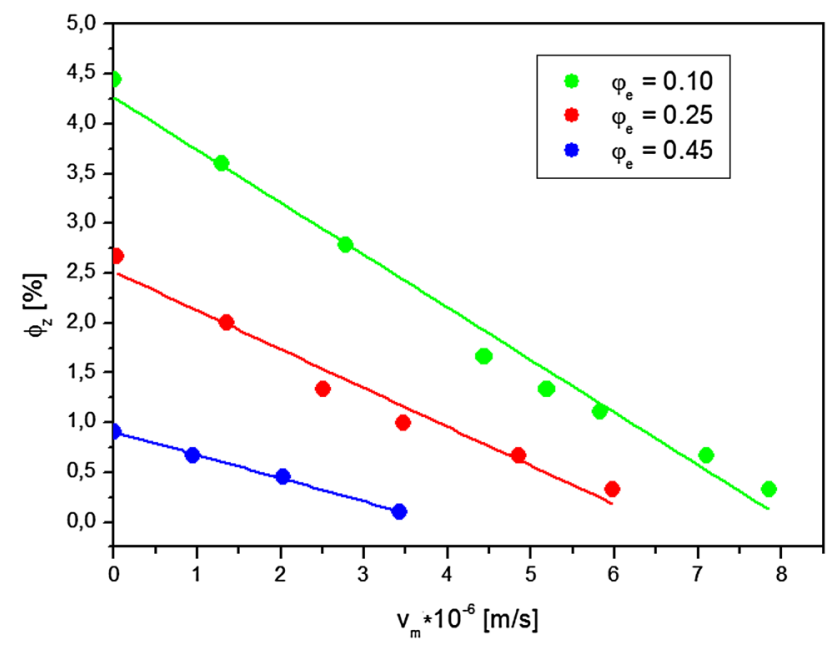

Fig. 7 Dependence of nanoparticle concentration in solution on mean velocity of emulsion destabilization for emulsions with different proportions of internal phase for an emulsion with a concentration of 0.45 vol. For each emulsion concentration, however, the dependence of creaming velocity on stabilizer concentration in each case can be described by a straight line.

The dependence of nanoparticle concentration on the average destabilization rate was also described by a linear relationship:

$\phi_{z}=A \cdot v_{\mathrm{m}}+B$,

where symbols $A$ and $B$ are the parameters of the straight line, where $A$ is the directional coefficient and $B$ is the intersection point with the $\phi_{z}$ axis. The values of these parameters are given in Table 1 for emulsion concentrations $\varphi_{\mathrm{e}} 0.1$; 0.25 and 0.45 vol.

The developed plot of the dependence of $\phi_{z}$ on $v_{\mathrm{m}}$, presented in Fig. 7, can be treated as standard curves for determining the concentration of nanoparticles in an unknown sample. For this purpose, it is necessary to prepare a Pickering emulsion with this sample in exactly the same way as the emulsions were created when preparing the standard curves. Such emulsion should then be subjected to destabilization analysis to determine the average velocity $v_{\mathrm{m}}$. Knowing the value of $v_{\mathrm{m}}$, it can be plotted against the standard curve and the nanoparticle concentration $\phi_{z}$ can be read off for a given emulsion concentration. Using a well-defined methodology for preparing emulsions and testing the destabilization process under the same conditions, it is possible to obtain results with an accuracy of $\pm 10 \%$, which has been confirmed experimentally. Taking into account the precision of even specialized equipment, such accuracy is satisfactory for practical use.

\section{Method limitations}

The basis for using the method to determine the concentration of nanoparticles in solution is the preparation of a suitable standard curve, i.e., the dependence of the concentration of the nanoparticles on the destabilization rate at a given emulsion concentration. The first limitation is that the nanoparticles under test must have the ability to form Pickering emulsions. If they exhibit such an ability, an appropriate

Table 1 Summaries of $A$ and $B$ parameters of relation (2) for different emulsion concentrations

\begin{tabular}{llll}
\hline $\begin{array}{l}\text { Emulsion concentra- } \\
\text { tion } \varphi_{\mathrm{e}}\end{array}$ & $A * 10^{-6}$ & $B$ & $R^{2}$ \\
\hline 0.10 & -0.5269 & 4.263 & 0.986 \\
0.25 & -0.3895 & 2.514 & 0.968 \\
0.45 & -0.2332 & 0.908 & 0.998 \\
\hline
\end{tabular}


choice of oil phase is necessary. The research presented here relates to a solution of nanoparticles in water. Experimental studies are needed to determine whether solutions with a solvent other than water will be applicable here. An important aspect is choosing the right concentration of the internal phase of the emulsion at which the systems are formed. The concentration of the emulsion must be chosen so that the destabilization process occurs with a given addition of nanoparticles. It must be remembered that above a certain concentration of nanoparticles, the emulsion remains stable and then the application of this method will not give results. Figure 8 shows the dependence of the concentration of silica nanoparticles in the aqueous phase on the concentration of emulsions prepared in the study at which the emulsion remains stable.

The curve of the dependence of $\phi_{z}$ on $\varphi_{\mathrm{e}}$, presented in Fig. 8, defines what are the limitations of using the method at a given emulsion concentration. If in an unknown sample the concentration of nanoparticles in solution is above the presented curve, the method will not give results. Therefore,

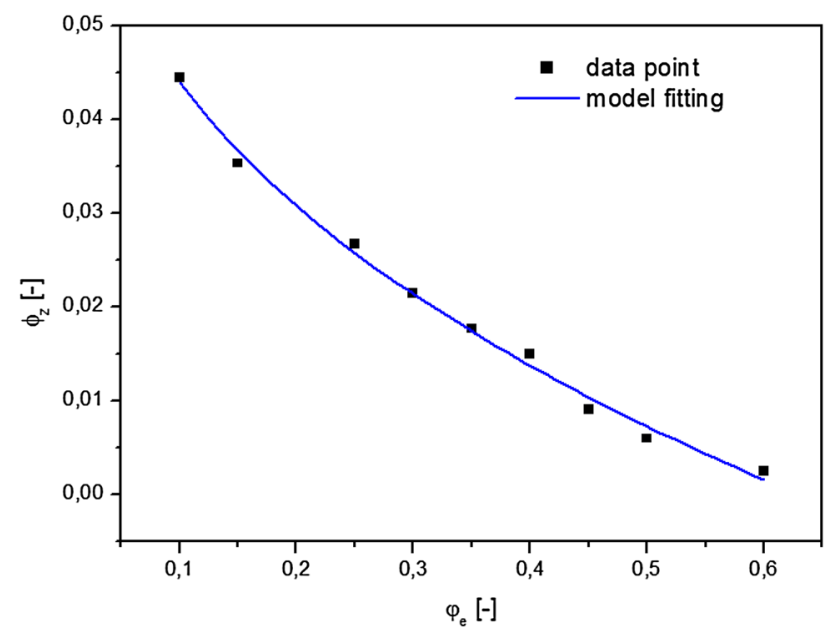

Fig. 8 Dependence of the concentration of nanoparticles in the aqueous phase on the concentration of the internal phase of the emulsion at which the emulsion remains stable the emulsion concentration should be chosen so that the amount of nanoparticles in solution is not so high as to form stable emulsions. As can be seen in Fig. 8, the lower the concentration of the internal phase, the higher the concentration of nanoparticles is needed to maintain full stability. Therefore, if higher concentrations of nanoparticles are expected in an unknown sample, emulsions with lower concentrations should be selected. On the other hand, if small amounts of nanoparticles are expected in solution, it is better to create emulsions at higher concentrations, as this can provide better accuracy of results.

\section{Example of application of the method for determination of nanoparticle concentration based on emulsion destabilization analysis}

To demonstrate how the presented method can be used in practice, we will illustrate an example of its application to determine the concentration of nanoparticles in solution obtained after diffusion. The experiments were performed using diffusion chambers (Franz chambers) to determine the rate of diffusion of nanoparticles through various membranes. After the diffusion process, an aqueous solution of silica nanoparticles of unknown concentration was obtained. Figure 9 shows a scheme of procedure for determining the concentration of nanoparticles in a sample according to the proposed method.

The volume of the sample was $20 \mathrm{ml}$. The first step was to prepare a Pickering emulsion using the sample. A comparative emulsion concentration of 0.25 vol. was chosen. While preparing the standard curve under the given conditions, an emulsion sample of $100 \mathrm{ml}$ was prepared in the same vessel with the same stirring rates and time. The emulsion sample with the unknown nanoparticle concentration also had to have the same volume at formation. Therefore, the unknown sample was first thoroughly mixed, using ultrasound, with $55 \mathrm{ml}$ of water and then mixed with $25 \mathrm{ml}$ of oil. The emulsification process was then carried out in the same manner

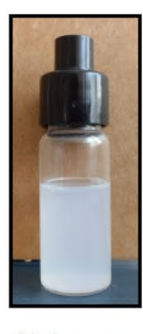

Unknown sample
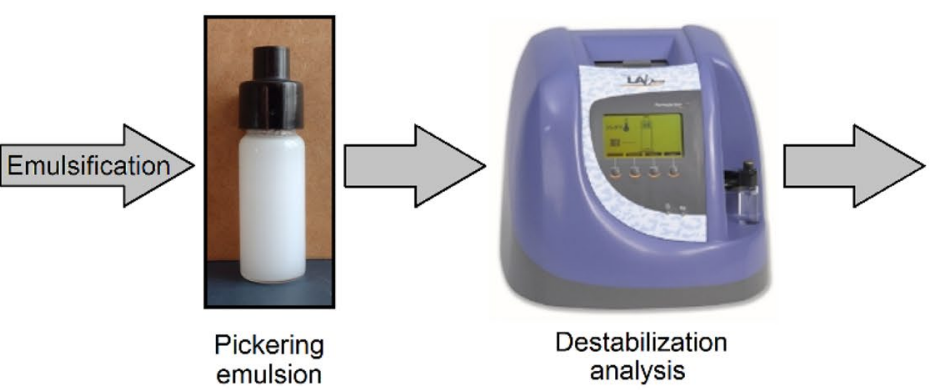

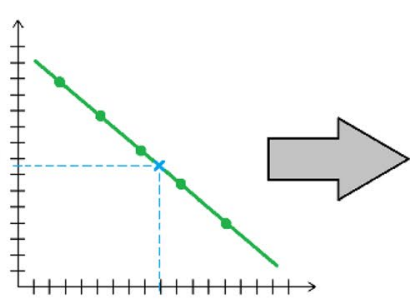

Reading from standard curve

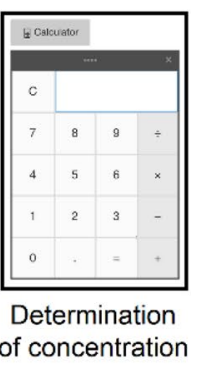

of concentration

Fig. 9 Scheme of procedure for determining the concentration of nanoparticles in solution 
a

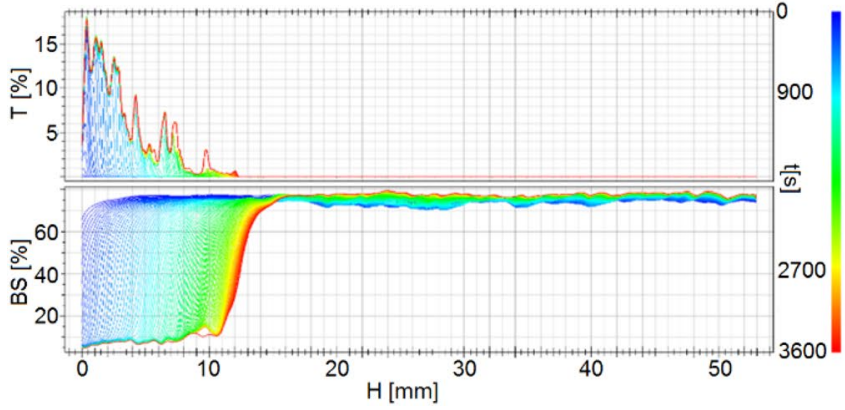

b

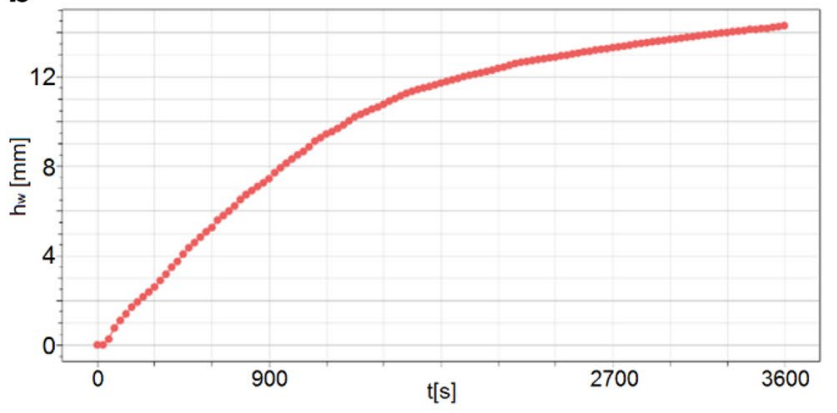

Fig. 10 Turbidimetric analyses of an emulsion prepared with an unknown nanoparticle sample: a transmittance and reflected light values with sample height, $\mathbf{b}$ increase in aqueous phase thickness during emulsion destabilization process

as in the formation of the standard curve. The emulsions produced were subjected to destabilization analysis for $1 \mathrm{~h}$ using Turbiscan. As a result of these analyses, changes in transmitted and reflected light along the sample height were obtained (Fig. 10a). From this, it was possible to estimate the changes in the height of the aqueous phase $h_{\mathrm{w}}$ (Fig. 10b). The final value (after $3600 \mathrm{~s}$ ) of $h_{\mathrm{w}}$, which was $14.2 \mathrm{~mm}$, has been read from the graph. This value was then divided by the duration of the process and the average velocity $v_{m}$ was obtained, which equaled $3.94 \cdot 10^{-6} \mathrm{~m} / \mathrm{s}$. Having this value, it can be plotted on the calibration curve graph (Fig. 7) or substituted under formula (2) for a concentration of $0.25 \mathrm{vol}$. and the value $\phi_{z}$ was calculated, which in the given example was $0.98 \%$. Note, however, that the read concentration $\phi_{z}$ refers to the concentration of particles in the aqueous phase of the emulsion $(100 \mathrm{ml})$. That is, in this case, we know what the concentration is in the nanoparticles in $75 \mathrm{ml}$ of water. However, we are interested in what the concentration was in the original $20 \mathrm{ml}$ sample. This is easy to calculate because the same proportion as in $75 \mathrm{ml}$ was found in $20 \mathrm{ml}$. So for our case, we find that the concentration of nanoparticles in the aqueous solution obtained after the diffusion process was $3.67 \%$.

\section{Summary and conclusions}

An important aspect is the ability to quantify the concentration of nanoparticles present in the solution. In this work, an indirect method to make such determinations has been proposed. This method was based on destabilization analyses of nanoparticle-stabilized emulsions. The relationship between the destabilization rate of such emulsions and their internal phase concentration and nanoparticle content was used. After a thorough investigation of the destablization mechanism, the relationship between the nanoparticle concentration and the destabilization rate of emulsions with a given internal phase concentration was described. This allowed the development of a standard curve from which the concentration of nanoparticle content in an unknown sample can be determined. An example of the application of such method is also presented, where the concentration of nanoparticles in an aqueous solution obtained after diffusion was determined. The presented method referred to silica nanoparticles, but a similar methodology can be used to determine the concentration of other nanoparticles having the ability to form Pickering emulsions. It should be added, that this method does not require a detailed ananlysis of the destabilization process. Only the average values of its velocity are necessary, which can be determined on the basis of simple optical techniques. The presented method gives satisfactory accuracy, especially considering that the results obtained with even expensive, high-tech equipment are associated with considerable error (Brown et al. 2013; Shang and Gao 2014; Merkus 2020). The paper also presents the limitations of the approach, but it should be emphasized that the advantages of this method are simplicity and lack of the need for specialized equipment. It is worth keeping in mind that despite the continuous development of measurement techniques, simple analytical methods are still in demand for economic reasons.

Supplementary Information The online version contains supplementary material available at https://doi.org/10.1007/s13204-021-02213-8.

Acknowledgements The study was financed within a research grant NCN Sonata 2019/35/D/ST8/01033

Funding The authors declare they have no financial interests.

\section{Declarations}

Conflict of interest None. 
Open Access This article is licensed under a Creative Commons Attribution 4.0 International License, which permits use, sharing, adaptation, distribution and reproduction in any medium or format, as long as you give appropriate credit to the original author(s) and the source, provide a link to the Creative Commons licence, and indicate if changes were made. The images or other third party material in this article are included in the article's Creative Commons licence, unless indicated otherwise in a credit line to the material. If material is not included in the article's Creative Commons licence and your intended use is not permitted by statutory regulation or exceeds the permitted use, you will need to obtain permission directly from the copyright holder. To view a copy of this licence, visit http://creativecommons.org/licenses/by/4.0/.

\section{References}

Alasonati E, Caebergs T, Pétry J, Sebaïhi N, Fisicaro P, Feltin N (2021) Size measurement of silica nanoparticles by asymmetric flow field-flow fractionation coupled to multi-angle light scattering: a comparison exercise between two metrological institutes. J Chrom A 1638:461859. https://doi.org/10.1016/j. chroma.2020.461859

Al-Thobity AM, Gad MM (2021) Effect of silicon dioxide nanoparticles on the flexural strength of heat-polymerized acrylic denture base material: a systematic review and meta-analysis. Saudi Dent J. https://doi.org/10.1016/j.sdentj.2021.08.008

Ashby N, Binks B (2000) Pickering emulsions stabilized by laponite clay particles. PCCP 2:5640-5646

Bao Y, Zhang Y, Liu P, Maa J, Zhang W, Liu C, Simion D (2019) Novel fabrication of stable Pickering emulsion and latex by hollow silica nanoparticles. J Coll Int Sci 553:83-90

Becher P (1957) Emulsions: theory and practice. Reinhold Publishing Corp, New York

Binks B, Lumsdon S (2001) Pickering emulsions stabilized by monodisperse latex particles: effects of particle size. Langmuir $17: 4540-4547$

Binks B, Yin D (2016) Pickering emulsions stabilized by hydrophilic nanoparticles: in situ surface modification by oil. Soft Matter 12:6858-6867

Brar SK, Verma M (2011) Measurement of nanoparticles by lightscattering techniques. TrAC 30(1):4-17. https://doi.org/10. 1016/j.trac.2010.08.008

Brown SC, Boyko V, Meyers G, Voetz M, Wohlleben W (2013) Toward advancing nano-object count metrology: a best practice framework. Env Health Persp 121:11-12. https://doi.org/ 10.1289/ehp. 1306957

Calabrese V, Courtenay JC, Edler KJ, Scott JL (2018) Pickering emulsions stabilized by naturally derived or biodegradable particles. Curr Opin Green Sustain Chem 12:83-90

Chao JB, Wang JR, Zhang JQ (2020) Accurate determination and characterization of gold nanoparticles based on single particle-inductively coupled plasma-mass spectrometry. Chinese J Anal Chem 48(7):946-954. https://doi.org/10.1016/S1872-2040(20)60032-9

Chevalier Y, Bolzinger M (2013) Emulsions stabilized with solid nanoparticles: Pickering emulsions. Coll Surf A Physicochem Eng Asp 439:23-34

Commission Recommendation of 18 October 2011 on the definition of nanomaterial (2011/696/EU)

Cunha AG, Mougel JB, Cathala B, Berglund LA, Capron I (2014) Preparation of double Pickering emulsions stabilized by chemically tailored nanocelluloses. Langmuir 30:9327-9335

Dammak I, dA Sobral PJ (2018) Formulation optimization of lechthin-enhanced Pickering emulsions stabilized by chitosan nanoparticles for hesperidin encapsulation. J Food Eng 229:2-11 du Sorbier MQ, Aimable A, Pagnoux C (2015) Influence of the electrostatic interactions in a Pickering emulsion polymerization for the synthesis of silica-polystyrene hybrid nanoparticles. J Coll Int Sci 448:306-314. https://doi.org/10.1016/j.jcis.2015.02.017

Eivazzadeh-Keihan R, Bahreinizad H, Amiri Z, Aliabadi HAM, Salimi-Bani M, Nakisa A, Davoodi F, Tahmasebi B, Ahmadpour F, Radinekiyan F, Maleki A, Hamblin MR, Mahdavi M, Madanchi H (2021) Functionalized magnetic nanoparticles for the separation and purification of proteins and peptides. TrAC 141:116291. https://doi.org/10.1016/j.trac.2021.116291

Gallego-Urrea JA, Tuoriniemi J, Hassellöv M (2011) Applications of particle-tracking analysis to the determination of size distributions and concentrations of nanoparticles in environmental, biological and food samples. TrAC 30(3):473-483. https://doi. org/10.1016/j.trac.2011.01.005

Jin A, Wang Y, Lin K, Jiang L (2020) Nanoparticles modified by polydopamine: working as "drug" carriers. Bioact Mater 5(3):522-541. https://doi.org/10.1016/j.bioactmat.2020.04.003

Kaur R, Bhardwaj SK, Chandna S, Kim KH, Bhaumik J (2021) Lignin-based metal oxide nanocomposites for UV protection applications: a review. J Clean Prod 317:128300. https://doi. org/10.1016/j.jclepro.2021.128300

Kegl T, Kralj AK, Kegl B, Kegl M (2021) Nanomaterials as fuel additives in diesel engines: a review of current state, opportunities, and challenges. Prog Energ Comb Sci 83:100897. https:// doi.org/10.1016/j.pecs.2020.100897

Khalegh R, Qaderi F (2019) Optimization of the effect of nanoparticle morphologies on the cost of dye wastewater treatment via ultrasonic/photocatalytic hybrid process. Appl Nanosci 9:1869_ 1889. https://doi.org/10.1007/s13204-019-00984-9

Khramtsov P, Kalashnikova T, Bochkova M, Kropaneva M, Timganova V, Zamorina S, Rayev M (2021) Measuring the concentration of protein nanoparticles synthesized by desolvation method: comparison of Bradford assay, BCA assay, hydrolysis/UV spectroscopy and gravimetric analysis. Inter J Pharm 599:120422. https://doi.org/10.1016/j.ijpharm.2021.120422

Kumar DK, Kř́ž J, Bennett N, Chen B, Upadhayaya H, Reddy KR, Sadhu V (2020) Functionalized metal oxide nanoparticles for efficient dye-sensitized solar cells (DSSCs): a review. Mater Sci Energ Technol 3:472-481. https://doi.org/10.1016/j.mset. 2020.03.003

Lebdioua K, Aimable A, Cerbelaud M, Videcoq A, Peyratout C (2018) Influence of different surfactants on Pickering emulsions stabilized by submicronic silica particles. J Coll Int Sci 520:127-133

Liao L, Zhang Z, Meng F, Liu D, Wu B, Li Y, Xie W (2021) A novel slurry for chemical mechanical polishing of single crystal diamond. Appl Surf Sci 564:150431. https://doi.org/10.1016/j. apsusc.2021.150431

Low LE, Siva SP, Hoa YK, Chan ES, Tey BT (2020) Recent advances of characterization techniques for the formation, physical properties and stability of Pickering emulsion. Adv Coll Int Sci 277:102-117

Menneveux J, Wang F, Lu S, Bai X, Motto-Ros V, Gilon N, Chen Y, Yu J (2015) Direct determination of Ti content in sunscreens with laser-induced breakdown spectroscopy: line selection method for high $\mathrm{TiO}_{2}$ nanoparticle concentration. Spectrochim Acta B Atom Spectr 109:9-15. https://doi.org/10.1016/j.sab.2015.04.010

Merkus HG (2020) Errors in particle size and concentration analysis of pharmaceuticals. Pharm Dev Technol 25(2):252-259. https:// doi.org/10.1080/10837450.2019.1690507

Metin CO, Rankin KM, Nguyen QP (2014) Phase behavior and rheological characterization of silica nanoparticle gel. Appl Nanosci 4:93-101. https://doi.org/10.1007/s13204-012-0168-7

Park J, Kwak M, Song NW et al (2020) Effect of colloidal nanoparticle concentration on sizing analysis with an electrospray scanning

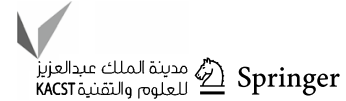


mobility particle sizer. Appl Nanosci 10:329-336. https://doi.org/ 10.1007/s13204-019-01115-0

Pellequer Y, Zanetta G, Rebibou JM et al (2021) Development of a new methodology to determine size differences of nanoparticles with nanoparticle tracking analysis. Appl Nanosci 11:2129-2141. https://doi.org/10.1007/s13204-021-01932-2

Pickering SU (1907) CXCVI-emulsions. J Chem Soc 91:2001-2021

Ramsden W (1903) Separation of solids in the surface-layers of solutions and suspensions (observations on surface-membranes, bubbles, emulsions, and mechanical coagulation)_preliminary account. Proc Roy Soc Lond 72:156-164

Shahbazi N, Zare-Dorabei R, Naghib SM (2021) Multifunctional nanoparticles as optical biosensing probe for breast cancer detection: a review. Mater Sci Eng C 127:112249. https://doi.org/10.1016/j. msec.2021.112249

Shang J, Gao X (2014) Nanoparticle counting: towards accurate determination of the molar concentration. Chem Soc Rev 43(21):72677278. https://doi.org/10.1039/c4cs00128a

Sheikholeslami Z, Ehteshami M (2020) Investigation of nanoparticle effect on performance of solar membrane distillation. Appl Nanosci. https://doi.org/10.1007/s13204-020-01560-2

Simovic S, Prestidge C (2004) Nanoparticles of varying hydrophobicity at the emulsion droplet-water interface: adsorption and coalescence stability. Langmuir 20:8357-8365

Sudagidan M, Yildiz G, Onen S, Al R, Temiz S, Yurt MNZ, Tasbasi BB, Acar EE, Coban A, Aydin A, Dursun AD, Ozalp VC (2021) Targeted mesoporous silica nanoparticles for improved inhibition of disinfectant resistant Listeria monocytogenes and lower environmental pollution. J Hazard Mater 418:126364. https://doi.org/ 10.1016/j.jhazmat.2021.126364

Tchami JH, Youmssi A, Kamta M, Gurlui S, Kayem GJ (2019) Real time determination in aqueous medium of the chemical nature and concentration of suspended nanoparticles produced by pulsed laser ablation using a simple infrared turbidimeter and an optical fiber probe. Opt Mater 95:109183. https://doi.org/10.1016/j. optmat.2019.06.009

Wang B, Zhang Z, Chang K, Cui J, Rosenkranz A, Yu J, Lin C-T, Chen G, Zang K, Luo J, Jiang N, Guo D (2018) New deformationinduced nanostructure in silicon. Nano Lett 18:4611-4617. https:// doi.org/10.1021/acs.nanolett.8b01910

Wang N, Dheen ST, Ying J, Fuh H, Kumar AS (2021) A review of multi-functional ceramic nanoparticles in 3D printed bone tissue engineering. Bioprinting 23:e00146. https://doi.org/10.1016/j. bprint.2021.e00146

Xie W, Zhang Z, Liao L, Liu J, Su H, Wang S, Guo D (2020) Green chemical mechanical polishing of sapphire wafers using a novel slurry. Nanoscale 12:22518-22526. https://doi.org/10.1039/ D0NR04705H
Yang Y, Fang Z, Chen X, Zhang W, Xie Y, Chen Y, Liu Z, Yuan W (2017) An overview of pickering emulsions: solid-particle materials, classification, morphology, and applications. Front Pharm 8:287. https://doi.org/10.3389/fphar.2017.00287

Yaraki MT, Tan YN (2020) Recent advances in metallic nanobiosensors development: colorimetric, dynamic light scattering and fluorescence detection. Sens Inter 1:100049. https://doi.org/10. 1016/j.sint1.2020.100049

Yu H, Guo Y, Zhu W, Havener K, Zheng X (2021a) Recent advances in 1,8-naphthalimide-based small-molecule fluorescent probes for organelles imaging and tracking in living cells. Coord Chem Rev 444:214019. https://doi.org/10.1016/j.ccr.2021.214019

Yu J, Wang AC, Zhang M, Linb Z (2021b) Water treatment via nonmembrane inorganic nanoparticles/cellulose composites. Mater Today. https://doi.org/10.1016/j.mattod.2021.03.024

Zhang Z, Song Y, Xu C, Guo D (2012) A novel model for undeformed nanometer chips of soft-brittle $\mathrm{HgCdTe}$ films induced by ultrafine diamond grits. Scr Mater 67(2):197-200. https://doi.org/10. 1016/j.scriptamat.2012.04.017

Zhang Z, Wang B, Kang R, Zhang B, Guo D (2015) Changes in surface layer of silicon wafers from diamond scratching. CIRP Ann 64(1):349-352. https://doi.org/10.1016/j.cirp.2015.04.005

Zhang Z, Cui J, Wang B, Wang Z, Kang R, Guo D (2017) A novel approach of mechanical chemical grinding. J Alloy Compd 726:514-524. https://doi.org/10.1016/j.jallcom.2017.08.024

Zhang Z, Shi Z, Du Y, Yu Z, Guo L, Guo D (2018) A novel approach of chemical mechanical polishing for a titanium alloy using an environment-friendly slurry. Appl Surf Sci 427:409-415. https:// doi.org/10.1016/j.apsusc.2017.08.064

Zhang Z, Cui J, Zhang J, Liu D, Yu Z, Guo D (2019) Environment friendly chemical mechanical polishing of copper. Appl Surf Sci 467-468:5-11. https://doi.org/10.1016/j.apsusc.2018.10.133

Zhang Z, Liao L, Wang X, Xie W, Guo D (2020) Development of a novel chemical mechanical polishing slurry and its polishing mechanisms on a nickel alloy. Appl Surf Sci 564(506):144670. https://doi.org/10.1016/j.apsusc.2019.144670

Zhang Z, Liu J, Hu W, Zhang L, Xie W, Liao L (2021) Chemical mechanical polishing for sapphire wafers using a developed slurry. J Manuf Proc 62:762-771. https://doi.org/10.1016/j.jmapro.2021. 01.004

Zheng K, Boccaccini AR (2017) Sol-gel processing of bioactive glass nanoparticles: a review. Adv Coll Inter Sci 249:363-373. https:// doi.org/10.1016/j.cis.2017.03.008

Publisher's Note Springer Nature remains neutral with regard to jurisdictional claims in published maps and institutional affiliations. 